$\xi=-1$

\title{
Monophonic wirelength on Circulant Networks
}

\author{
K. Uma Samundesvari ${ }^{1 *}$, Sindhuja G. Michael $^{2}$ \\ ${ }^{1}$ Assistant Professor, Department of Mathematics, Noorul Islam Center for Higher Education, Thuckalay-629 180, Kanyakumari,India \\ ${ }^{2}$ Assistant Professor, Department of Mathematics, Ponjesly College of Engineering, Nagercoil, 629003, India \\ *Corresponding author E-mail:kuskrishna@gmail.com
}

\begin{abstract}
In this paper, we define the monophonic embedding of graph $G$ into graph $H$ and we present an algorithm for finding the monophonic wirelength of circulant networks into the family of grids $M(n \times 2), n \geq 2$. The monophonic embedding of a graph $G$ into a graph $H$ is an embedding denoted by $f_{m}$ is a bijective map from the vertex set of $G$ into the vertex set of $H$ and $f_{m}$ is a one-one mapping from the edge set $(x, y)$ of $G$ into $P_{m}(H)$ where $P_{m}(H)$ is the set of monophonic paths between $f_{m}(x)$ and $f_{m}(y)$ for every $f_{m}(x), f_{m}(y) \in H$. The monophonic wirelength of $f_{m}$ of $G$ into $H$ is the sum of distances of monophonic paths between two vertices $f_{m}(x)$ and $f_{m}(y)$ in $H$ such that $(x, y) \in$ $E(G)$. This paper presents a monophonic algorithm to find the monophonic wirelength of circulant networks $G(2 n, \pm S)$, where $S \subseteq$ $\{1,2,3, \ldots, n\}$ into the family of grids $\mathrm{M}[\mathrm{n} \times 2], n \geq 2$. We also derived a Lemma to get the monophonic edge congestion $M E C(G, H)$.
\end{abstract}

Keywords: Circulant Networks; Edge Congestion; Grid; Monophonic Distance; Wirelength.

\section{Introduction}

The distance $\mathrm{d}(\mathrm{x}, \mathrm{y})$ between two vertices $\mathrm{x}$ and $\mathrm{y}$ in a graph $\mathrm{G}$ is the length of the shortest path from $x$ to $y$ in $G$. An edge $x_{i} x_{j}$ is a chord of a path $\mathrm{x}_{0}, \mathrm{x}_{1}, \mathrm{x}_{2}, \ldots, \mathrm{x}_{\mathrm{n}}$ if $\mathrm{j} \geq \mathrm{i}+2$. A monophonic path is a path if it contains no chord. The length of the longest $x-y$ monophonic path of a graph $G$ is called the monophonic distance $\mathrm{d}_{\mathrm{m}}(\mathrm{x}, \mathrm{y})$ for every vertices $\mathrm{x}, \mathrm{y}$ in $\mathrm{G}$. A monophonic path from $\mathrm{x}$ to $\mathrm{y}$ with length $\mathrm{d}_{\mathrm{m}}(\mathrm{x}, \mathrm{y})$ is called an $\mathrm{x}-\mathrm{y}$ monophonic "as stated in $[1,2,3,4]$ ". Consider a graph $\mathrm{H}$, since other graphs or networks are embedded into it, as host graph and graphs or networks which are embedded in $\mathrm{H}$ are called guest graph "as given in $[5,6,7]$ ". Let $\mathrm{G}(V, E)$ and $H(V, E)$ be finite graphs with $n$ vertices. An embedding $\mathrm{f}$ of $\mathrm{G}$ into $\mathrm{H}$ is defined as follows:

1) $f$ is a bijective map from $\mathrm{V}(\mathrm{G}) \rightarrow \mathrm{V}(\mathrm{H})$

2) $f$ is a one-to-one map from $\mathrm{E}(\mathrm{G})$ to $\left\{\mathrm{P}_{\mathrm{f}}(f(\mathrm{u}), f(\mathrm{v})): \mathrm{P}_{\mathrm{f}}(\mathrm{f}(\mathrm{u})\right.$, $\mathrm{f}(\mathrm{v}))$ is a path in $\mathrm{H}$ between $f(\mathrm{u})$ and $f(\mathrm{v})$ for $(\mathrm{u}, \mathrm{v}) \in \mathrm{E}(\mathrm{G})\}$ "as defined in $[8,9]$. i.e., The embedding $f$ of $\mathrm{G}$ to $\mathrm{H}$ is a bijective mapping from the vertex set of $G$ to the vertex set of $\mathrm{H}$ and every edge (u, v) $\in \mathrm{E}(\mathrm{G})$ is mapped to a path between $f(\mathrm{u})$ and $f(\mathrm{v})$ in $\mathrm{H}$. The edge congestion of an embedding $f$ of $\mathrm{G}$ into $\mathrm{H}$ is the maximum number of edges of the graph $\mathrm{G}$ that are embedded on any single edge of $\mathrm{H}$. The wirelength of an embedding $f$ of $\mathrm{G}$ into $\mathrm{H}$ is given by

$W L_{f}(G, H)=\sum_{(u, v) \in E(G)} d_{H}(f(u), f(v))=\sum_{e \in E(H)} E C_{f}(G, H(e))$

If we find an embedding of $\mathrm{G}$ into $\mathrm{H}$ which produces the minimum wirelength WL $(G, H)$, such problem is called the wirelength problem "as stated in [9]". We use definitions, Lemmas and Theorems from [1], [2], [5], [6], [7], [8] and [9] for this work.

\section{Monophonic wirelength problem}

Definition 2.1: Let $G(V, E)$ and $H(V, E)$ be finite graphs with $n$ vertices. An embedding $f_{m}: G \rightarrow H$ is called a monophonic embedding if $f_{m}$ maps each vertex of $G$ into a vertex of $H$ and each edge $(x, y)$ of $G$ is mapped to a monophonic path between $f_{m}(x)$ and $f_{m}(y)$ in $H$.

Definition 2.2: Let $f_{m}: G \rightarrow H$ be a monophonic embedding. The monophonic edge congestion of $f_{m}$ of $G$ into $H$ is the maximum number of edges of the graph $G$ that are embedded on an edge $e \in H$ and is given by

$M E C_{f_{m}}(G, H)=\max M E C_{f_{m}}(G, H(e))$

The monophonic wirelength problem of a graph $\mathrm{G}$ into $\mathrm{H}$ is the problem of finding a monophonic embedding $f_{\mathrm{m}}: \mathrm{G} \rightarrow \mathrm{H}$ that produces the monophonic wire length $\mathrm{MW} \mathrm{L}(\mathrm{G}, \mathrm{H})$.

Definition 2.3: Let $f_{m}: G \rightarrow H$ be a monophonic embedding. The monophonic wirelength $M W L(G, H)$ of $f_{m}$ is given as

$$
M W L_{f_{m}}(G, H)=\sum_{(x, y) \in E(G)} d_{m}\left(f_{m}(x), f_{m}(y)\right)
$$

Proposition 2.4: For embeddings $f: G \rightarrow H$ and the monophonic embeddings $f_{m}: G \rightarrow H, W L_{f}(G, H) \leq M W L_{f m}(G, H)$

Proof: From Lemma 2.3 "as proved in [2]", we have

$$
\sum_{(x, y) \in E(G)} d_{H}(f(x), f(y)) \leq \sum_{(x, y) \in E(G)} d_{m}\left(f_{m}(x), f_{m}(y)\right)
$$

And using definitions "as defined in [9]" we write, 


$$
\begin{aligned}
W L_{f}(G, H) & =\sum_{(x, y) \in E(G)} d_{H}(f(x), f(y)) \\
& =\sum_{e \in E(H)} E C_{f}(G, H(e)) \\
& \leq \sum_{(x, y) \in E(G)} d_{m}\left(f_{m}(x), f_{m}(y)\right) \\
& =M W L_{f_{m}}(G, H)
\end{aligned}
$$

Therefore,

$$
W L_{f}(G, H) \leq M W L_{f_{m}}(G, H)
$$

Lemma 2.5: (Monophonic congestion Lemma)Let $G$ be an $r$ regular graph with $n$ vertices. Let $H$ be a finite graph with $n$ vertices. Let $f_{m}: G \rightarrow H$ be a monophonic embedding of $G$ into $H$. Let the graph $H \backslash E_{j}, j=1,2, \ldots, p ; 0<p \leq|E(G)|$, have the components $H_{i}, i=1,2$ and $G_{i}=f_{m}^{-1}\left(H_{i}\right)$, where $E_{j}$ 's are the edge cuts of $H$, form a partition in $H$ and have the following properties:

i) For $\mathrm{m} \geq 0$, there are $\mathrm{m}$ edges $(\mathrm{x}, \mathrm{y}) \in \mathrm{G}_{\mathrm{i}}, \mathrm{i}=1,2$; such that the monophonic path $\boldsymbol{P}_{f_{m}}\left(f_{m}(x), f_{m}(y)\right)$ has exactly two edges in $E_{j}$.

ii) The monophonic path $P_{f_{m}}\left(f_{m}(x), f_{m}(y)\right)$ has exactly one edge in $E_{\mathrm{j}}$ for every $(\mathrm{x}, \mathrm{y}) \in \mathrm{G}$ with $\mathrm{x} \in \mathrm{G}_{1} \& \mathrm{y} \in \mathrm{G}_{2}$ where $\mathrm{G}_{1}$ is the maximum subgraph in G. Then $\operatorname{MEC}_{f_{m}}\left(E_{j}\right)$ is monophonic and the monophonic wirelength of $f_{\mathrm{m}}$ of $\mathrm{G}$ into $\mathrm{H}$ is given by

$M W L_{f m}(G, H)=\sum_{j=1}^{p} M E C_{f m}\left(E_{j}\right)$

Where, $\operatorname{MEC}_{f m}\left(E_{j}\right)=r|V(G 1)|-2|E(G 1)|+2, m \geq 0$.

Proof: As $\mathrm{E}_{\mathrm{j}}, \mathrm{j}=1,2, \ldots, \mathrm{p}$ are edge cuts of $\mathrm{H}$ $E_{j}=\left\{(u, v) \in E(H) ; u \in H_{1}, v \in H_{2}\right\}$.

Let $T=\left\{(x, y) \in E(G) ; x \in G_{1}, y \in G_{2}\right\}$. Since there are ' $\mathrm{m}$ ' edges $(\mathrm{x}, \mathrm{y}) \in \mathrm{G}_{\mathrm{i}}, \mathrm{i}=1,2$; the monophonic path $\boldsymbol{P}_{f_{m}}\left(f_{m}(x), f_{m}(y)\right)$ in $\mathrm{H}$ has exactly two edges in $\mathrm{E}_{\mathrm{j}}$. Therefore, the monophonic edge congestion is increased by $2 \mathrm{~m}$ from the edge congestion of $\mathrm{E}_{\mathrm{j}}$. Also the monophonic path $P_{f_{m}}\left(f_{m}(x), f_{m}(y)\right)$

exactly one edge in $\mathrm{E}_{\mathrm{j}}$ for every

$M E C_{f^{\prime}}\left(E_{j}\right)=|T+2 m|$.

Where $|\mathrm{T}|=\mathrm{r}\left|\mathrm{V}\left(\mathrm{G}_{1}\right)\right|-2 \mid \mathrm{E}\left(\mathrm{G}_{1}\right)$ by Lemma 2 in [9].

Therefore, $\mathrm{EC}_{\mathrm{fm}}$ is monophonic as $\mathrm{G}_{1}$ is maximum in $\mathrm{G}$. The edge cuts $E_{j}, j=1,2$, $p$ form a partition in $\mathrm{H}$. Thus, we write

$$
M W L_{f m}(G, H)=\sum_{j=1}^{p} M E C_{f m}\left(E_{j}\right)
$$

\section{Monophonic wirelength on circulant net- works}

Definition 3.1:A circulant undirected graph denoted by $G(n, \pm S)$ where $S \subseteq\{1,2,3, \ldots,[n / 2]\}, n \geq 3$ is defined as a graph consisting of the vertex set $V=\{0,1,2, \ldots, n-1\}$ and the edge set $E=\{(i, j): \mid i$ $j \mid \equiv s(\bmod n), s \in S\}$ " as defined in [5]".

To present the monophonic wirelength on circulant networks, we consider the monophonic embedding $f_{m}$ from the circulant graph $G[2 n, \pm S], S \subseteq\{1,2,3 \ldots n\}$, into the grid $M(n \times 2), n \geq 2$.

\subsection{Monophonic algorithm}

Consider the monophonic embedding $f_{\mathrm{m}}: \mathrm{G}[2 \mathrm{n}, \pm \mathrm{S}] \rightarrow \mathrm{M}[\mathrm{n} \times 2]$. Let $V(G[2 n, \pm S])=\{0,1,2 \ldots 2 n-1\}$ and these vertices are labeled as the vertices of a cycle in clockwise.Let $\mathrm{V}(\mathrm{M}[\mathrm{n} \times 2])=\{0$, $1,2 \ldots 2 \mathrm{n}-1\}$, these vertices are named as follows

- In Column 1 of $M[n \times 2]$ the vertices $\{0,1,2 \ldots n-1\}$ are named in an ascending order from the top.

- In Column 2 of $M[n \times 2]$ the vertices $\{n, n+1 \ldots, 2 n-1\}$ are named in an ascending order from the top.

Lemma 3.2:For $n \geq 2$, the rows of the grid $M[n \times 2]$ is defined as $R_{i}=\{i-1, n+i-1\} ; i=1,2, \ldots, n$ are maximum subgraphs in $G[2 n ;\{1,2, \ldots, n\}]$.

The proof holds by Theorems 3.3 and 3.4 "as proved in [6]".

Lemma 3.3: For $j=1$, and $n \geq 2$, the column of the grid $M[n \times 2]$ is given by $C_{j}=\{0,1,2, \ldots, n-1\}$, which is maximum in $G[2 n ;\{1,2, \ldots, n\}]$.

The proof follows from Theorem 3.3 and 3.4 "as proved in [6]".

Theorem 3.4:Let $f_{m}: G[2 n, \pm S] \rightarrow M[n \times 2]$ be a monophonic embedding. For $n \geq 2$, the wirelength of $G[2 n, \pm S]$,

$S \subseteq\{1,2 \ldots, n\}$ into $M[n \times 2]$ induced by $f_{m}$ is monophonic.

Proof: Let $A_{i 1}$ and $A_{i 2}$ be the components of $M[n \times 2] \backslash H_{i}, H_{i}$ be the horizontal edge cut of the grid $M[n \times 2]$.Then the vertex set of $A_{i 1}$ is the rows of the component $A_{i 1}$. (ie) $V\left(A_{i 1}\right)$ is $R_{i}, i$ $=1,2, \ldots, 2 \mathrm{n}-1$. Refer Figure 1 (a). Let $\mathrm{B}_{\mathrm{j} 1}$ and $\mathrm{B}_{\mathrm{j} 2}$ be the components of $M[n \times 2] \backslash W_{j}, W_{j}$ be the vertical edge cut of the grid $M[n \times 2]$. Then the vertex set of $\mathrm{B}_{\mathrm{j} 1}$ is $\mathrm{C}_{\mathrm{j}}, \mathrm{j}=1$. Under $f_{\mathrm{m}}$ let $\mathrm{G}_{\mathrm{i} 1}=$ $\boldsymbol{f}_{m}^{-1}\left(\boldsymbol{A}_{i 1}\right)$ and $\mathrm{G}_{\mathrm{i} 2}=f_{m}^{-1}\left(A_{i 2}\right)$. Since the horizontal edge cuts satisfy the properties stated in Lemma 2.5 and are maximum in $\mathrm{G}$, $\operatorname{MEC}_{f_{m}}\left(\boldsymbol{H}_{i}\right)$ is monophonic for $\mathrm{i}=1,2, \ldots 2 \mathrm{n}-1$. For this see Figure 1(b). Let $\mathrm{G}_{\mathrm{j} 1}=f_{m}^{-1}\left(\boldsymbol{B}_{j 1}\right)$ and $\mathrm{G}_{\mathrm{j} 2}=f_{m}^{-1}\left(\boldsymbol{B}_{j 2}\right)$. Moreover the vertical edge cut $\mathrm{W}_{\mathrm{j}}$ satisfies the properties stated in Lemma 2.5. Also by Lemma 3.3, $\mathrm{G}_{\mathrm{j} 1}$ is a maximum sub graph induced by the vertices of $\mathrm{C}_{\mathrm{j}}, \mathrm{j}=1$. Hence by Lemma 2.5, $M E C_{f_{m}}\left(W_{j}\right)$ is monophonic for $\mathrm{j}=1$. Thus $\boldsymbol{M E C}_{f_{m}}(\boldsymbol{G}, \boldsymbol{H})$ is monophonic.

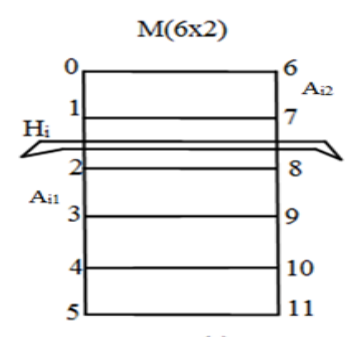

(a)
Figure 1

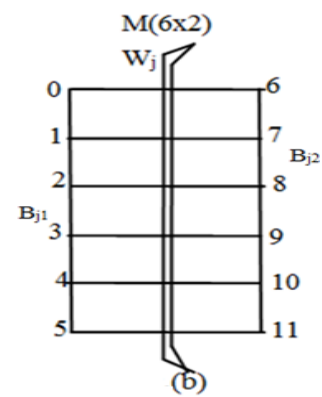

Fig. 1:(a) Each $H_{i}$ is an edge cut of the grid on $M[6 \times 2]$ which disconnects $M[6 \times 2]$ into two components $A_{i 1}$ and $A_{i 2}$ where $V\left(A_{i 1}\right)$ Is $R_{i}$. (b) Each $W_{j}$ is an edge cut of the grid on $M[6 \times 2]$ which disconnects $M[6 \times 2]$ into two components $\mathrm{B}_{\mathrm{j} 1}$ and $\mathrm{B}_{\mathrm{j} 2}$ where $\mathrm{V}\left(\mathrm{B}_{\mathrm{j} 2}\right)$ is $\mathrm{C}_{\mathrm{j}}$.

Theorem 3.5:The monophonic wirelength of an r-regular graph $G$ with $2 n$ vertices into the grid $M[n \times 2], n \geq 2$ is given by $M W L(G$, $M[n \times 2])=W L(G, M[n \times 2])+2 m$

Proof: Let $\mathrm{f}_{\mathrm{m}}: \mathrm{G} \rightarrow \mathrm{M}[\mathrm{n} \times 2$ ] be a monophonic embedding. Since each edge $(\mathrm{x}, \mathrm{y})$ of $\mathrm{G}$ is mapped to a monophonic path between $f_{\mathrm{m}}(\mathrm{x})$ and $f_{\mathrm{m}}(\mathrm{y})$ in $\mathrm{M}[\mathrm{n} \times 2]$, the edges of $\mathrm{G}$ are transformed into edges vertically or horizontally in $\mathrm{M}[\mathrm{n} \times 2]$. Therefore there exists horizontal edge cuts $\mathrm{H}_{\mathrm{i}}, \mathrm{i}=1,2, \ldots, 2 \mathrm{n}-1$ in $\mathrm{M}[\mathrm{n} \times 2]$. As there are no monophonic paths $\boldsymbol{P}_{f_{m}}\left(f_{m}(x), f_{m}(y)\right)$, for every $(\mathrm{x}, \mathrm{y}) \in \mathrm{G}_{\mathrm{i}}$, i $=1,2$; having edges in $\mathrm{H}_{\mathrm{i}}$, the monophonic edge congestion of $\mathrm{H}_{\mathrm{i}}$ is equivalent to the edge congestion of the edges of $\mathrm{H}_{\mathrm{i}}$.Also there exists a vertical edge cut $\mathrm{W}_{\mathrm{j}}, \mathrm{j}=1$. For $\mathrm{m} \geq 0$, there are $\mathrm{m}$ edges 
$(\mathrm{x}, \mathrm{y}) \in \mathrm{G}_{\mathrm{i}}, \mathrm{i}=1,2$; the monophonic paths

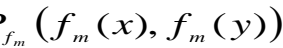

have exactly two edges in $\mathrm{W}_{\mathrm{j}}$ and so the monophonic edge congestion of the edges of $\mathrm{W}_{\mathrm{j} i s}$ increased by $2 \mathrm{~m}$ from the edge congestion of the edges of $\mathrm{W}_{\mathrm{j}}$. Hence the monophonic wirelength of each row equals the wirelength of each row of $M[n \times 2]$ and the monophonic wirelength of each column differs by $2 \mathrm{~m}$ from the wirelength of columns of $M[n \times 2]$.

Therefore $\operatorname{MWL}(\mathrm{G}, \mathrm{M}[\mathrm{n} \times 2])=\mathrm{WL}(\mathrm{G}, \mathrm{M}[\mathrm{n} \times 2])+2 \mathrm{~m}$

Theorem 3.6: $M W L(G[2 n, \pm 1], M[n \times 2])=W L(G[2 n, \pm 1]$, $M[n \times 2])=2(2 n-1)$.

Proof: As there is no edge $(a, b) \in G_{i}, i=1,2$; the monophonic path $\boldsymbol{P}_{f_{m}}\left(f_{m}(\boldsymbol{a}), \boldsymbol{f}_{m}(\boldsymbol{b})\right) \in M[n \times 2]$ has exactly two edges in $E_{j}, m=0$. Therefore, the result follows from Theorem 3.5 .

\section{Monophonic embedding algorithm}

\subsection{Aim}

To find a monophonic embedding $f_{\mathrm{m}}: \mathrm{G} \rightarrow \mathrm{H}$ that produces the monophonic wirelength $\boldsymbol{M W L}(\boldsymbol{G}, \boldsymbol{H})$, where G is the family of circulant graph with $2 \mathrm{n}$ vertices of r-regular and $\mathrm{H}$ is the family of grid $M[n \times 2], n \geq 2$.

\subsection{Monophonic algorithm}

i) Label the vertices of $G[2 n,\{1,2,3 \ldots, n-1\}]$ as a cycle from $0,1,2, \ldots, 2 \mathrm{n}-1$

ii) Label the vertices of $M[n \times 2]$ as follows:

- In Column 1 of $M$ [n×2] the vertices $\{0,1,2, n-1\}$ are labeled in an ascending order from the top.

- In Column 2 of $M[n \times 2]$ the vertices $\{n, n+1 \ldots, 2 n-1\}$ are labeled in an ascending order from the top.

Case (i): Input:

Pre-image: The family of circulant graph G[2n, $\{1,2, n-1\}], n \geq 2$. Image: The family of grids $M[n \times 2] n \geq 2$.

Output: A monophonic embedding $\mathrm{f}_{\mathrm{m}}$ of $\mathrm{G}[2 \mathrm{n},\{1,2,3 \ldots, \mathrm{n}-1\}]$ into $\mathrm{M}(\mathrm{n} \times 2)$ given by $f_{\mathrm{m}}(\mathrm{x})=\mathrm{x}$ with monophonic wire length $\operatorname{MWL}(\mathrm{G}[2 \mathrm{n},\{1,2,3, \ldots, \mathrm{n}-1\}], \mathrm{M}[\mathrm{n} \times 2])=\mathrm{WL}+2(\mathrm{n}-1)(\mathrm{n}-2), \mathrm{n} \geq 2$.

Case (ii): Input:

Pre-image: The family of circulant graphs G[2n, $\{1,2, n-2\}], n \geq 3$. Image: The family of grids $M[n \times 2], n \geq 3$.

Output: A monophonic embedding $f_{\mathrm{m}}$ of $\mathrm{G}[2 \mathrm{n},\{1,2,3 \ldots, \mathrm{n}-2\}]$ into $\mathrm{M}[\mathrm{n} \times 2]$ given by $f_{\mathrm{m}}(\mathrm{x})=\mathrm{x}$ with monophonic wire length $\operatorname{MWL}(G[2 n,\{1,2,3, \ldots, n-2\}], M[n \times 2])=W L+2 n(n-3), n \geq 3$.

Case (iii): Input:

Pre-image: The family of circulant graphs G $[2 n,\{1,2, \ldots, n-4\}], n$ $\geq 5$.

Image: The family of grids $M[n \times 2], n \geq 5$.

Output: A monophonic embedding $\mathrm{f}_{\mathrm{m}}$ of $\mathrm{G}[2 \mathrm{n},\{1,2,3 \ldots, \mathrm{n}-4\}]$ into $\mathrm{M}[\mathrm{n} \times 2]$ given by $f_{\mathrm{m}}(\mathrm{x})=\mathrm{x}$ with monophonic wire length $\operatorname{MWL}(G[2 n,\{1,2,3, \ldots, n-4\}], M[n \times 2])=W L+2(n+2)(n-5), n \geq 5$.

Case (iv): Input:

Pre-image: The family of circulant graphs $G[2 n,\{1,2, \ldots, n\}], n$ $\geq 2$

Image: The family of grids $M[n \times 2], n \geq 2$.

Output: A monophonic embedding $\mathrm{f}_{\mathrm{m}}$ of $\mathrm{G}[2 \mathrm{n},\{1,2,3 \ldots, \mathrm{n}\}]$ into $\mathrm{M}[\mathrm{n} \times 2]$ given by $f_{\mathrm{m}}(\mathrm{x})=\mathrm{x}$ with monophonic wire length $\operatorname{MWL}(\mathrm{G}[2 \mathrm{n},\{1,2,3, \ldots, \mathrm{n}\}], \mathrm{M}[\mathrm{n} \times 2])=\mathrm{WL}+2(\mathrm{n}-1)(\mathrm{n}-2), \mathrm{n} \geq 2$.

Proof: For all the above Cases (i) to (iv), using Theorem 3.4, the mapping $f_{m}$ is monophonic and by Theorem 3.5 , the results follows.

\section{Conclusion}

In this paper, we applied the monophonic idea on graph embed$\operatorname{ding} f$ of two graphs from $\mathrm{G}$ into $\mathrm{H}$. Using this concept; we have obtained a modified result in the wirelengthproblem, which does not exist. A new technique was found from the existing one. We have taken all possible family of circulant networks under study and applied the monophonic algorithm on $f$ and based on the statistical data we obtained we came to the conclusion which yields the above findings.

\section{References}

[1] Buckley F \&Harary F, "Distance in Graphs", Addison-Wesley, Redwood City, CA, (1990).

[2] Santhakumaran AP \& Titus P, "Monophonic distance in graphs", Discrete Math, Algorithms and Appl., Vol.3, No.2, (2011), pp.159169.https://doi.org/10.1142/S1793830911001176.

[3] Maya P \& Nicholas T, "Some Results on Integer Edge Cordial Graph", DJ Journal of Engineering and Applied Mathematics, Vol.3, No.1, (2017), pp.12.https://doi.org/10.18831/djmaths.org/2017011001.

[4] Mathew J, "Subdivided Stars Super (b, d)-Edge-Antimagic Total Graph Labelling", DJ Journal of Engineering and Applied Mathematics, Vol.1, No.1, (2015), pp.3034.https://doi.org/10.18831/djmaths.org/2015011005.

[5] Rajasingh I, Manuel P, Arockiaraj M \&Rajan B, "Embeddings of Circulant networks", Journal of Combinatorial Optimization, Vol.26, No.1, (2011), pp.135-151.https://doi.org/10.1007/s10878 011-9443-X

[6] Quadras J \& Surya SS, "Linear wirelength of circulant networks", International Journal of Pure and Applied Mathematics, Vol.86, No.6, (2013), pp.883-891.

[7] Arokiaraj M, Manuel P, Rajasingh I \&Rajan B, "Wirelength of 1fault Hamiltonian graphs into wheels and fans",Information Processing Letters, Vol.111, (2011) pp.921925.https://doi.org/10.1016/j.ipl.2011.06.011

[8] .Bezrukov SL, Chavez JD, Harper LH, Rottger M \& Schroeder UP, "Embedding of hypercubes into grids", MFCS, Electronic Edition Springer, Lecture Notes in Computer Science, No.1450, (1998), pp.693-701.https://doi.org/10.1007/BFb0055820.

[9] Manuel P, Rajasingh I, Rajan B \& Mercy H, "Exact wirelength of hypercube on a grid", Discrete Applied Mathematics, Vol.157, No.7, (2009), pp.1486 1495.https://doi.org/10.1016/j.dam.2008.09.013. 